\title{
ORDER ADAPTATION OF THE FRACTIONAL FOURIER TRANSFORM USING THE INTRAFRAME PITCH CHANGE RATE FOR SPEECH RECOGNITION
}

\author{
Hui Yin ${ }^{1,2}$, Climent Nadeu ${ }^{2}$, Volker Hohmann ${ }^{2,3}$, Xiang Xie $^{1}$, Jingming Kuang ${ }^{1}$ \\ 1. Dept. of Electronic Engineering, Beijing Institute of Technology, Beijing \\ 2. TALP Research Center, Universitat Politècnica de Catalunya, Barcelona \\ 3. Medical Physics, Universität Oldenburg
}

\begin{abstract}
We propose an acoustic feature for speech recognition based on the combination of MFCC and fractional Fourier transform (FrFT). The transform orders for FrFT are adaptively set according to the intraframe pitch change rate. This method is motivated by the fact that the speech is not stationary even in a short period of time, and the idea is shown using an AM-FM speech model and some spectrograms of an artificial periodic signal. Experiments were conducted on the intervocalic English consonants provided by Interspeech 2008 Consonant Challenge and a Mandarin connected digits corpus. The performance of the proposed method is compared with the MFCC baseline system. Experimental results show that the proposed features get a slightly better recognition rate than MFCCs presumably because they can better track the dynamic characteristics of the speech harmonics.
\end{abstract}

Index Terms - fractional Fourier transform, pitch, feature extraction, speech recognition, consonant challenge

\section{INTRODUCTION}

Speech is a non-stationary signal. Traditional speech processing methods generally treat speech as short-time stationary, i.e., process speech in $20 \sim 30 \mathrm{~ms}$ frames. Melfrequency cepstral coefficients (MFCC), which are the most widely used features for automatic speech recognition (ASR), also rely on the short-time stationarity assumption. MFCCs model speech across frequency and reflect the frequency characteristics of the human auditory system.

In practice, intonation and coarticulation introduce combined spectro-temporal fluctuations to speech even for the typical frame sizes used in the front-end analysis. Modeling speech signals as frequency modulation signals accords better with speech characteristics from both production and perception views. From the speech production view, traditional linear source-filter theory lacks the ability to explain the refined structure of speech in a pitch period. In 1980s Teager discovered by experiments that vortices could be the secondary source to excite the channel and produce the speech signal. Therefore, speech should be composed of the plane-wave-based linear part and a vortices-based non-linear part [1]. According to such theory, Maragos et al. proposed an AM-FM modulation model for speech analysis, synthesis and coding. The AMFM model represents the speech signal as the sum of formant resonance signals each of which contains amplitude and frequency modulation [2]. From the perception view, neurophysiological studies show that the auditory system of mammals is sensitive to FM-modulated (chirpy) sounds. This fact explains the human hearing sensitivity to nonstationary acoustic events with changing pitch (police and ambulance siren) [3].

Fractional Fourier transform can be considered as a generalization of the traditional Fourier transform. In 1980, Namias first introduced the mathematical definition of the FrFT [4]. Then, Almeida analyzed the relationship between the FrFT and the Wigner-Ville Distribution (WVD), and interpreted it as a rotation operator in the time-frequency plane. Since FrFT can be considered as a decomposition of the signal in terms of chirps, FrFT is especially suitable for the processing of chirp-like signals [5].

When using FrFT, the determination of the optimal transform order is always critical. The order is a free parameter of FrFT directly related with the chirp rate, i.e. the temporal derivative of instantaneous frequency, of the signal. Since voiced speech has a harmonic structure, setting the order of the FrFT to be some pitch-based value might improve the representation of the time-varying properties of speech. This approach is investigated in this study and evaluated by consonant and digit recognition experiments.

The rest of the paper is organized as follows. In section 2, the AM-FM model of speech is described, and the motivation of the proposed method is given. In section 3 , the definition and some basic properties of FrFT are briefly introduced. In section 4 , the determination of the pitchbased orders and the FrFT-MFCC feature extraction method is given. Section 5 presents the ASR experimental results and discussion. Some conclusions are given in section 6 .

\section{THE AM-FM MODEL OF SPEECH}

In speech recognition, coding and synthesis, pitch and formants are always the most important parameters. In the 
traditional speech processing method, these parameters are considered as constant within a frame. Since voiced speech has a harmonic structure, it can be modeled as:

$$
x(t)=\sum_{n=1}^{\infty} a_{n}(t) \cos \left(n \omega_{0} t+\theta_{n}\right)
$$

where $a_{n}(t)$ is time-varying amplitude signal, $\omega_{0}$ is the pitch, $\theta_{n}$ is the initial phase, $\mathrm{n}$ is the number of the harmonics.

But in fact, the pitch is always changing even within a frame because of the tonation. Considering the fluctuation of pitch and the harmonic structure, speech can be modeled as an AM-FM signal

$$
x(t)=\sum_{n=1}^{\infty} a_{n}(t) \cos \left(n\left(\omega_{0} t+\int_{0}^{t} q(\tau) d \tau\right)+\theta_{n}\right),
$$

where $q(t)$ is the frequency modulation function. Making a reasonable simplification that the frequency is changing linearly within this frame, i.e., $q(t)=k t$ with $k$ being the chirp rate of the pitch (referred to as pitch rate in the rest of the paper), we can obtain:

$$
x(t)=\sum_{n=1}^{\infty} a_{n}(t) \cos (\underbrace{n\left(\omega_{0} t+\frac{1}{2} k t^{2}\right)+\theta_{n}}_{\varphi_{n}(t)})
$$

The chirp rate of the Nth harmonic is the second derivative of the phase function $\frac{d^{2} \varphi_{N}(t)}{d t^{2}}=q_{N}=N k$, which means the chirp rate of the Nth harmonic is also $\mathrm{N}$ times of the pitch rate.

\section{DEFINITION OF THE FRACTIONAL FOURIER TRANSFORM}

The FrFT of signal $x(t)$ is represented as:

$$
X_{\alpha}(u)=F_{p}[x(t)]=\int_{-\infty}^{\infty} x(t) K_{\alpha}(t, u) d t,
$$

where $p$ is a real number and called the order of the FrFT, $\alpha=p \pi / 2$ is the transform angle, $F_{p}[\bullet]$ denotes the FrFT operator, and $K_{\alpha}(t, u)$ is the kernel of the FrFT:

$$
K_{\alpha}(t, u)=\left\{\begin{array}{lr}
\sqrt{\frac{1-j \cot \alpha}{2 \pi}} \exp \left(j \frac{t^{2}+u^{2}}{2} \cot \alpha-j u t \csc \alpha\right), \\
\alpha \neq n \pi \\
\delta(t-u), & \alpha=2 n \pi \\
\delta(t+u), & \alpha=(2 n \pm 1) \pi
\end{array}\right.
$$

The kernel has the following properties:

$$
\begin{aligned}
& K_{-\alpha}(t, u)=K_{\alpha}^{*}(t, u) \\
& \int_{-\infty}^{\infty} K_{\alpha}(t, u) K_{\alpha}^{*}\left(t, u^{\prime}\right) d t=\delta\left(u-u^{\prime}\right)
\end{aligned}
$$

Hence, the inverse FrFT is

$$
x(t)=F_{-p}\left[X_{\alpha}(u)\right]=\int_{-\infty}^{\infty} X_{\alpha}(u) K_{-\alpha}(t, u) d u
$$

Eq.(8) indicates that signal $x(t)$ can be interpreted as a decomposition to a basis formed by the orthonormal Linear Frequency Modulated (LFM) functions in the $u$ domain, which means an LFM signal with a chirp rate corresponding to the transform order $p$ can be transformed into an impulse in a certain fractional domain. Therefore, the FrFT has excellent localization performance for LFM signals.

\section{ACOUSTIC FEATURE EXTRACTION}

\subsection{Pitch and pitch rate calculation}

The speech is processed in overlapping frames. Each frame is further divided into several non-overlapping sub-frames. One pitch value is detected for one subframe. These pitch values are obtained using a robust pitch tracking algorithm described in [6] and implemented in Matlab. In order to get the chirp rate of a frame, we first calculate the median value of the sub-frame pitch values for this frame to set a threshold, if any sub-frame pitch value is larger than twice this threshold, then it is divided by 2 . If any pitch value is smaller than half the threshold, it is multiplied by 2 . In this way, octave or sub-octave confusions are largely eliminated. Then, linear curve fitting is applied to fit all the corrected pitch values in this frame. The pitch rate is taken as the slope of this fitted line. For unvoiced speech, the transform order will be 1 because no pitch is detected.

\subsection{Transform order determination}

In order to emphasize the Nth harmonic with the chirp rate $\mathrm{N} * \mathrm{k}$, the transform angle of the FrFT is chosen as

$$
\alpha=\operatorname{acot}(-2 \pi * k * N) \text {. }
$$

In order to illustrate the effect of emphasizing harmonics, we produced a pulse train with linearly decreasing fundamental frequency and plotted its FFT-based spectrogram and FrFT-based spectrogram with $\mathrm{N}=10$ in Figure 1. It can be seen that the 10th harmonic and its neighbors are emphasized, i.e. they have better concentration performance than the FFT-based spectrogram. On the other hand, the representation of those harmonics whose chirp rate deviates from the chirp rate that corresponds to the selected transform order (low harmonics in this example) is smeared. 


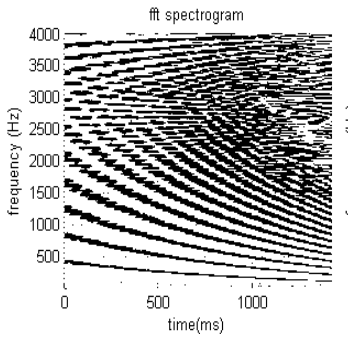

(a)

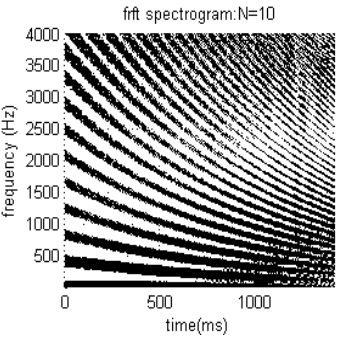

(b)
Figure 1: The FFT and FrFT based spectrogram of frequencyvarying pulse train. (a) is the result using FFT, and (b) is the result using FrFT with orders corresponding to 10 times of the pitch rates

\subsection{Feature extraction}

The proposed feature extraction consists of the same processing used for the MFCCs but substituting the Fourier transform (FT) by FrFT (denoted as FrFT-MFCC, see a block diagram in Figure 2). The sub-band energies that are usually employed to compute the speech recognition features, e.g. in the widely used mel frequency cepstral coefficients (MFCC), are a representation of the envelope, i.e. the formant structure, of voiced speech. Therefore, the aim of the mel-scale sub-band integration (and also the truncation of the sequence of cepstral coefficients in the MFCC representation) is to make the harmonic structure disappear in order to have a pitch-free envelope representation. Nevertheless, the FT-based spectral harmonics are an intermediate step in the computation of the envelope, so a more precise representation of the harmonics in relevant regions of the spectral envelope may help to get more discriminative speech features that improve the speech recognition rate.

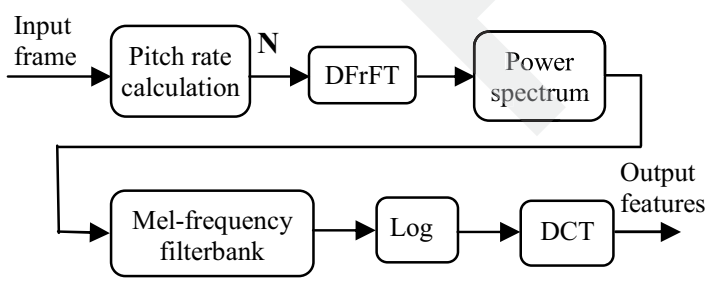

Figure 2: Diagram of the FrFT-MFCC extraction

\section{EXPERIMENTAL RESULTS}

\subsection{Speech corpus}

The experiments were conducted on two different corpora. One is the intervocalic consonants (VCV) provided by the Interspeech 2008 Consonant Challenge [7]. Twelve female and 12 male native English talkers produced each of the 24 consonants(/b/, /d/, /g/, /p/, /t/, /k/, /s/, /sh/, /f/, /v/, /th/, /dh/, /ch/, /z/, /zh/, /h/, /dz/, /m/, /n/, /ng/, /w/, /r/, /y/, /1/) in nine vowel contexts consisting of all possible combinations of the three vowels /i:/, /u:/, and /ae/. The VCVs are sampled at $25 \mathrm{kHz}$, with 16 bit quantization.

Training material comes from 8 male and 8 female speakers while tokens from the remaining 8 speakers are used in the independent test set. After removing unusable tokens identified during post-processing, the training set consists of 6664 clean tokens.

We combined the 7 test sets as one large test set. For this, the clean speech signals were taken from the two-channel material that contains speech in one channel and noise in the other channel. Each of the 7 test sets contains 16 instances of each of the 24 consonants, for a total of $384 * 7$ tokens in the combined test set.

The other corpus is composed of Mandarin connected digits, with 3 5 connected digits in each utterance. 1117 utterances are used for training and other 891 utterances for recognition [9].

\subsection{Experiment design}

The baseline is the same as in the Consonant Challenge. Speech is analyzed using a frame length of $25 \mathrm{~ms}$ and a frame shift of $10 \mathrm{~ms}$. The speech is parameterized with 12 MFCC coefficients and log energy and augmented with first and second temporal derivatives resulting in a 39dimensional feature vector. Each of the monophones consists of 3 emitting states with a 24-Gaussian mixture output distribution. No silence model and short pause model are employed in this distribution as features are end-pointed. The HMMs were trained from a flat start using HTK. Cepstral mean normalisation (CMS) is used [8]. The same parameters and configurations described above are used to test FrFT-MFCC. Five subframes are used to detect the pitch values within one frame.

\subsection{Experimental results}

The transform orders for FrFT are set to be corresponding to $\mathrm{N}$ times of the calculated pitch rate. $\mathrm{N}$ is taken to be $1,2,3$, and 5. The recognition results are given in Table 1. Figure 3 depicts error rates for individual consonants using MFCC and the absolute improvement of every consonant over MFCC when using FrFT-MFCC with different N. The result using the ambiguity-function (AF)-based order search is also given just for comparison [9].

Table 1: Consonant Error Rates using different $N$ and $A F$ (\%)

\begin{tabular}{cccccc}
\hline FFT & $\mathrm{N}=1$ & $\mathrm{~N}=2$ & $\mathrm{~N}=3$ & $\mathrm{~N}=5$ & $\mathrm{AF}$ \\
\hline 15.29 & 14.66 & 14.40 & 15.07 & 15.10 & 14.99 \\
\hline
\end{tabular}




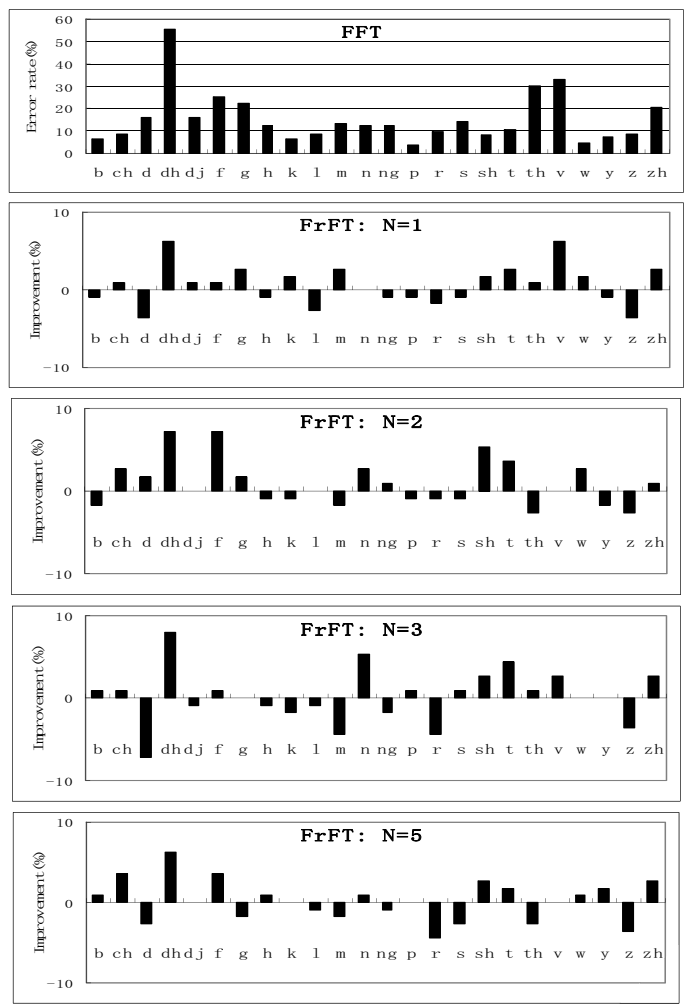

Figure 3: Error rates and improvements for each consonant

From Table 1, we can see the FrFT-MFCC with $\mathrm{N}=1,2,3,5$ all outperformed traditional MFCC. The best result is obtained when $\mathrm{N}=2$. When $\mathrm{N}$ gets larger, the formants may be dispersed because the chirp rates of formants is generally lower than $\mathrm{N}$ times the pitch rate. $\mathrm{N}=2$ can obtain a good compromise between tracking the dynamic speech harmonics and preserving the concentration of the formants.

From Figure 3, we can see that three consonants always encounter most errors: the dental fricatives, $/ \mathrm{dh}, \mathrm{th} /$, and the labiodentals fricatives $/ \mathrm{v} /$, just the same as in human listening test. These sounds were responsible for a large number of production errors. Different consonants achieve their peak performance at different $\mathrm{N}$. The improvements of the most error-prone consonants $/ \mathrm{dh} /, / \mathrm{f}, \mathrm{v} /$ are also most significant. Most voiced consonants achieve the best performance at $\mathrm{N}=1 \sim 3$, including: /g/, /zh/, /v/, /m/, /dj/, /w/, $/ \mathrm{d} /, / \mathrm{ng} /, / \mathrm{dh} /, / \mathrm{b} /$, and $/ \mathrm{n} /$; just for $/ \mathrm{y} /$, the best performance is at $\mathrm{N}=5$; for $/ \mathrm{z} /, / 1 /$ and $/ \mathrm{r} /$, FFT performs best.

The recognition results for Mandarin connected digits are given in Table 2 .

Table 2: Word Error Rates using different $N$ and $A F(\%)$

\begin{tabular}{cccccc}
\hline FFT & $\mathrm{N}=1$ & $\mathrm{~N}=2$ & $\mathrm{~N}=3$ & $\mathrm{~N}=5$ & $\mathrm{AF}$ \\
\hline 5.61 & 5.44 & 5.42 & 5.36 & 5.72 & 5.21 \\
\hline
\end{tabular}

From Table 2, we can see the proposed features also get slightly lower word error rates than MFCCs. The best result is obtained when $\mathrm{N}=3$. The AF-based method obtains a comparable result with the best result.

\section{CONCLUSIONS}

In this paper, we propose an acoustic feature set for speech recognition, which is a combination of MFCC and FrFT. The transform orders for FrFT are adaptively set according to the intraframe pitch change rate. When the order is set corresponding to $\mathrm{N}$ times of the pitch rate, $\mathrm{N}$ has an optimal value which can obtain a good compromise between tracking the dynamic characteristics of the speech harmonics and avoiding much dispersion of the formants within a frame. Compared to the order search method based on ambiguity function, the pitch-based method has much less computation complexity because the AF-based method is still a step search method. The disadvantage of the pitch determined order is that it relies greatly on the accuracy of pitch detection, which is a tough problem in noisy environment.

\section{ACKNOWLEDGEMENTS}

The research was supported in part by National Nature Science Foundation of China under Grant NSFC 60605015 and by a research grant to V.H. from the Spanish ministry of education and science.

\section{REFERENCES}

[1] H. M. Teager and S. M. Teager, "Evidence for Nonlinear Sound Production Mechanisms in the Vocal Tract", NATO Advanced Study Institute on Speech Production and Speech Modelling, Bonas, France, July 1989.

[2] P. Maragos, T. Quatieri, and J. F. Kaiser, "On Amplitude and Frequency Demodulation Using Energy Operators," IEEE Transaction on Acoustics, Speech and Signal Processing, 41(4), pp. 1532-1550, April 1993.

[3] M. K'epesi, L. Weruaga, "High-resolution noise-robust spectral-based pitch estimation", Interspeech, Lisbon, Portugal, 313-316, 2005.

[4] Namias V. The fractional order Fourier transform and its application to quantum mechanics. J Inst Math Appl, 25, 1980, 241-265.

[5] Qi Lin, Tao Ran, Zhou Si-yong, "Detection and parameter estimation of multicomponent LFM signal based on the fractional Fourier transform", Science in China, 47(2), 184-198, 2004

[6] D. Talkin, "A Robust Algorithm for Pitch Tracking (RAPT)" in "Speech Coding \& Synthesis", W B Kleijn, K K Paliwal eds, Elsevier ISBN 0444821694, 1995.

[7] The website of consonant challenge in Interspeech 2008: http://www.odettes.dds.nl/challenge IS08/material.html

[8] Martin Cooke, Odette Scharenborg, "The Interspeech 2008 consonant challenge", Interspeech, Brisbane, Australia, 2008.

[9] Yin Hui, Xie Xiang, Kuang Jingming, "Adaptive-Order Fractional Fourier Transform Features for Speech Recognition", Interspeech, Brisbane, Australia, 2008 